\title{
Analysis of the masses and decay constants of the heavy-light mesons with QCD sum rules
}

\author{
Zhi-Gang Wang ${ }^{\mathrm{a}}$ \\ Department of Physics, North China Electric Power University, Baoding 071003, People's Republic of China
}

Received: 24 July 2015 / Accepted: 28 August 2015 / Published online: 15 September 2015

(C) The Author(s) 2015. This article is published with open access at Springerlink.com

\begin{abstract}
In this article, we calculate the contributions of the vacuum condensates up to dimension- 6 including the $\mathcal{O}\left(\alpha_{s}\right)$ corrections to the quark condensates in the operator product expansion, then we study the masses and decay constants of the pseudoscalar, scalar, vector, and axial-vector heavy-light mesons with the QCD sum rules in a systematic way. The masses of the observed mesons $\left(D, D^{*}\right),\left(D_{s}, D_{s}^{*}\right)$, $\left(D_{0}^{*}(2400), D_{1}(2430)\right),\left(D_{s 0}^{*}(2317), D_{s 1}(2460)\right),\left(B, B^{*}\right)$, $\left(B_{s}, B_{s}^{*}\right)$ can be well reproduced, while the predictions for the masses of $\left(B_{0}^{*}, B_{1}\right)$ and $\left(B_{s 0}^{*}, B_{s 1}\right)$ can be confronted with the experimental data in the future. We obtain the decay constants of the pseudoscalar, scalar, vector, and axial-vector heavylight mesons, which have many phenomenological applications in studying the semi-leptonic and leptonic decays of the heavy-light mesons.
\end{abstract}

\section{Introduction}

The charged heavy-light mesons can decay to a charged lepton pair $\ell^{+} v_{\ell}$ through a virtual $W^{+}$boson. Those leptonic decays are excellent subjects in studying the CKM matrix elements and serve as a powerful probe of new physics beyond the standard model in a complementary way to the direct searches. For example, the decay widths of the pseudoscalar (P) and vector (V) heavy-light mesons can be written as

$$
\begin{aligned}
\Gamma(P \rightarrow \ell v)= & \frac{G_{F}^{2}}{8 \pi} f_{P}^{2} m_{\ell}^{2} m_{P}\left(1-\frac{m_{\ell}^{2}}{m_{P}^{2}}\right)^{2}\left|V_{q_{1} q_{2}}\right|^{2}, \\
\Gamma(V \rightarrow \ell \nu)= & \frac{G_{F}^{2}}{12 \pi} f_{V}^{2} m_{V}^{3}\left(1-\frac{m_{\ell}^{2}}{m_{V}^{2}}\right)^{2} \\
& \times\left(1+\frac{m_{\ell}^{2}}{2 m_{V}^{2}}\right)\left|V_{q_{1} q_{2}}\right|^{2},
\end{aligned}
$$

\footnotetext{
a e-mail: zgwang@aliyun.com
}

in the lowest order approximation, where $m_{P / V}$ and $f_{P / V}$ are the masses and decay constants, respectively, $m_{\ell}$ is the $\ell$ mass, $V_{q_{1} q_{2}}$ is the CKM matrix element between the constituent quarks $q_{1} \bar{q}_{2}$, and $G_{F}$ is the Fermi coupling constant. If we take the CKM matrix element $V_{q_{1} q_{2}}$ and the branching fractions of the leptonic decays from the CLEO, BaBar, Belle collaborations as input parameters, then the average values $f_{D}=(204.6 \pm 5.0) \mathrm{MeV}, f_{D_{s}}=(257.5 \pm 4.6) \mathrm{MeV}$, and $f_{D_{s}} / f_{D}=1.258 \pm 0.038$ are obtained [1]. It is difficult to reproduce the three values consistently in theoretical calculations, such as the QCD sum rules [2-7] and lattice QCD [8-11]. The discrepancies between the theoretical values and experimental data maybe signal some new physics beyond the standard model [12]. In Ref. [13], we observe that, if we take into account the $\mathcal{O}\left(\alpha_{s}^{2}\right)$ corrections to the perturbative terms and the $\mathcal{O}\left(\alpha_{s}\right)$ corrections to the quark condensate terms and choose the pole masses, the predictions $f_{D}=(211 \pm 14) \mathrm{MeV}, f_{D_{s}}=(258 \pm 13) \mathrm{MeV}$, and $f_{D_{s}} / f_{D}=1.22 \pm 0.08$ are in excellent agreement with the experimental data [1].

In the QCD sum rules for the heavy-light mesons, the Wilson coefficients of the vacuum condensates at the operator product expansion side from different references differ from each other in one way or the other according to the different approximations [2-4,13-21]. In this article, we recalculate the contributions of the vacuum condensates up to dimension-6, including the one-loop corrections to the quark condensates, and we take into account the terms neglected in previous work; then we study the masses and decay constants of the pseudoscalar, scalar, vector, and axial-vector heavy-light mesons in a systematic way.

There has been much theoretical work on the decay constants of the heavy-light mesons, such as the QCD sum rules [2-7, 12-32], the lattice QCD [8-11,33-38], the BetheSalpeter equation $[39,40]$, the relativistic potential model [41-43], the field-correlator method [44], the light-front quark model [45-47], the chiral extrapolation [48], the 
extended chiral-quark model $[49,50]$, the constituent quark model [51], etc.

The article is arranged as follows: we derive the QCD sum rules for the masses and decay constants of the heavy-light mesons in Sect. 2; in Sect. 3, we present the numerical results and discussions; and Sect. 4 is reserved for our conclusions.

\section{QCD sum rules for the heavy-light mesons}

In the following, we write down the two-point correlation functions $\Pi_{0 / 5}(p)$ and $\Pi_{V / A}^{\mu \nu}(p)$ in the QCD sum rules,

$$
\begin{aligned}
\Pi_{0 / 5}(p) & =i \int \mathrm{d}^{4} x \mathrm{e}^{i p \cdot x}\left\langle 0\left|T\left\{J_{0 / 5}(x) J_{0 / 5}^{\dagger}(0)\right\}\right| 0\right\rangle, \\
\Pi_{V / A}^{\mu \nu}(p) & =i \int \mathrm{d}^{4} x \mathrm{e}^{i p \cdot x}\left\langle 0\left|T\left\{J_{V / A}^{\mu}(x) J_{V / A}^{\nu \dagger}(0)\right\}\right| 0\right\rangle, \\
J_{0}(x) & =\bar{Q}(x) q(x) \\
J_{5}(x) & =\bar{Q}(x) i \gamma_{5} q(x), \\
J_{V}^{\mu}(x) & =\bar{Q}(x) \gamma^{\mu} q(x), \\
J_{A}^{\mu}(x) & =\bar{Q}(x) \gamma^{\mu} \gamma_{5} q(x),
\end{aligned}
$$

where the currents $J_{5}(x), J_{0}(x), J_{V}^{\mu}(x)$, and $J_{A}^{\mu}(x)$ interpolate the pseudoscalar, scalar, vector, and axial-vector heavylight mesons, respectively, $Q=c, b$ and $q=u, d, s$. We can insert a complete set of intermediate hadronic states with the same quantum numbers as the current operators $J_{5}(x), J_{0}(x)$, $J_{V}^{\mu}(x)$, and $J_{A}^{\mu}(x)$ into the correlation functions $\Pi_{0 / 5}(p)$ and $\Pi_{V / A}^{\mu \nu}(p)$ to obtain the hadronic representation $[52,53]$. After isolating the ground state contributions from the pseudoscalar, scalar, vector, and axial-vector heavy-light mesons, we get the following results:

$$
\begin{aligned}
\Pi_{0}(p) & =\frac{f_{S}^{2} m_{S}^{2}}{m_{S}^{2}-p^{2}}+\cdots, \\
\Pi_{5}(p) & =\frac{f_{P}^{2} m_{P}^{4}}{\left(m_{Q}+m_{q}\right)^{2}\left(m_{P}^{2}-p^{2}\right)}+\cdots, \\
\Pi_{V / A}^{\mu \nu}(p) & =\frac{f_{V / A}^{2} m_{V / A}^{2}}{m_{V / A}^{2}-p^{2}}\left(-g_{\mu \nu}+\frac{p^{\mu} p^{v}}{p^{2}}\right)+\cdots \\
& =\Pi_{V / A}(p)\left(-g_{\mu \nu}+\frac{p^{\mu} p^{v}}{p^{2}}\right)+\cdots
\end{aligned}
$$

where the decay constants $f_{S / P / V / A}$ are defined by

$$
\begin{aligned}
\left\langle 0\left|J_{0}(0)\right| S(p)\right\rangle & =f_{S} m_{S}, \\
\left\langle 0\left|J_{5}(0)\right| P(p)\right\rangle & =\frac{f_{P} m_{P}^{2}}{m_{Q}+m_{q}}, \\
\left\langle 0\left|J_{V / A}^{\mu}(0)\right| V / A(p)\right\rangle & =f_{V / A} m_{V / A} \epsilon^{\mu},
\end{aligned}
$$

the $\epsilon^{\mu}$ are the polarization vectors of the vector and axialvector mesons.

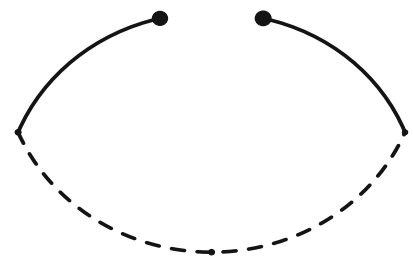

Fig. 1 The diagram contributes to the quark condensate $\langle\bar{q} q\rangle$

Now we carry out the operator product expansion at large space-like region $P^{2}=-p^{2}$. The analytical expressions of the perturbative $\mathcal{O}\left(\alpha_{s}\right)$ corrections to the perturbative terms for all the correlation functions [27,28] and the semi-analytical expressions of the perturbative $\mathcal{O}\left(\alpha_{s}^{2}\right)$ corrections to the perturbative terms for the pseudoscalar current's correlation functions [29,30] are available now. We take into account those analytical and semi-analytical expressions directly [27-30]; and we recalculate the contributions of the vacuum condensates, i.e. we calculate the Feynman diagrams shown in Figs. 1, 2, 3, 4, and 5, where the solid and dashed lines denote the light and heavy quark lines, respectively, and the wave line denotes the gluon line. In calculating the diagrams in Fig. 2, we correct the minor errors in Ref. [13], where the quark condensate $\frac{\langle\bar{q} q\rangle}{12}$ in the full lightquark propagators is replaced with $\frac{\langle\bar{q} q\rangle}{3 D}, D$ is the dimension of the space-time. A minor error occurs when there exist divergences, such a step should be deleted, i.e. the quark condensate $\frac{\langle\bar{q} q\rangle}{12}$ survives in the D-dimension. In Ref. [54], we correct the minor errors and improve the calculations, and we obtain the correct expressions. Furthermore, we obtain the perturbative $\mathcal{O}\left(\alpha_{s}\right)$ corrections to the quark condensate terms for the vector and axial-vector currents.

Once analytical expressions of the QCD spectral densities are obtained, then we can take the quark-hadron duality below the continuum thresholds and perform the Borel transforms with respect to the variable $P^{2}=-p^{2}$ to obtain the QCD sum rules,

$$
\begin{aligned}
\frac{f_{P}^{2} m_{P}^{4}}{\left(m_{Q}+m_{q}\right)^{2}} \exp \left(-\frac{m_{P}^{2}}{T^{2}}\right) & =B_{T} \Pi_{5}, \\
f_{S}^{2} m_{S}^{2} \exp \left(-\frac{m_{S}^{2}}{T^{2}}\right) & =B_{T} \Pi_{0}, \\
f_{V}^{2} m_{V}^{2} \exp \left(-\frac{m_{V}^{2}}{T^{2}}\right) & =B_{T} \Pi_{V}, \\
f_{A}^{2} m_{A}^{2} \exp \left(-\frac{m_{A}^{2}}{T^{2}}\right) & =B_{T} \Pi_{A},
\end{aligned}
$$

where

$$
\begin{aligned}
& B_{T} \Pi_{5}=B_{T} \Pi_{5}^{0}+B_{T} \Pi_{5}^{3}+B_{T} \Pi_{5}^{4}+B_{T} \Pi_{5}^{5}+B_{T} \Pi_{5}^{6}, \\
& B_{T} \Pi_{0}=\left.B_{T} \Pi_{5}\right|_{m_{Q} \rightarrow-m_{Q}},
\end{aligned}
$$



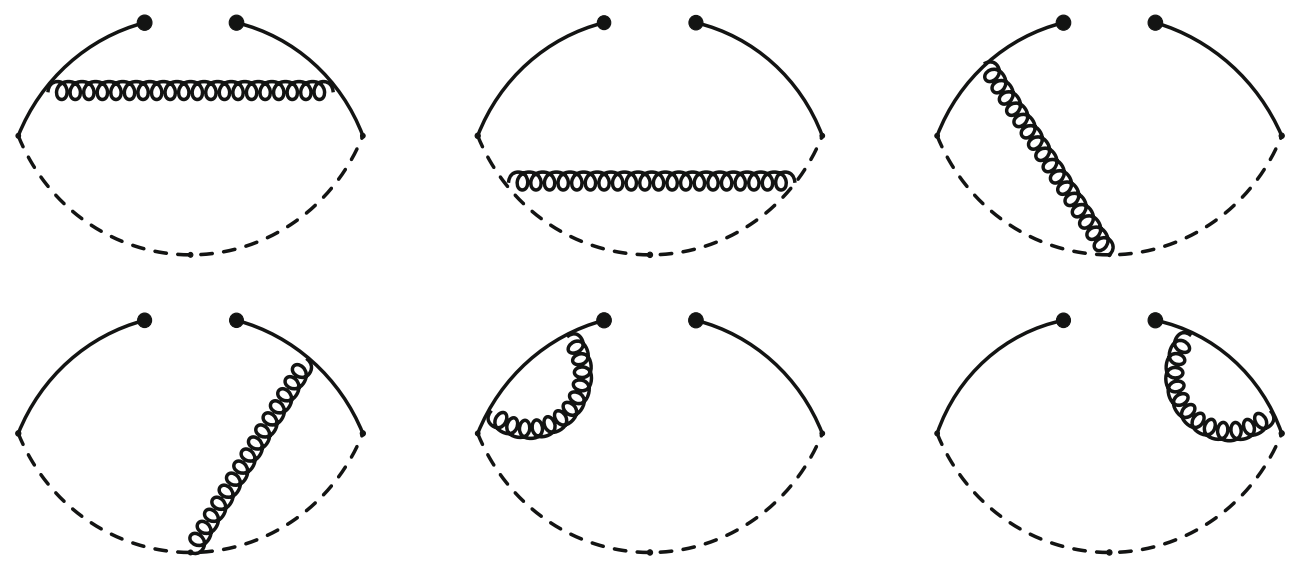

Fig. 2 The perturbative $\mathcal{O}\left(\alpha_{s}\right)$ corrections to the quark condensate $\langle\bar{q} q\rangle$
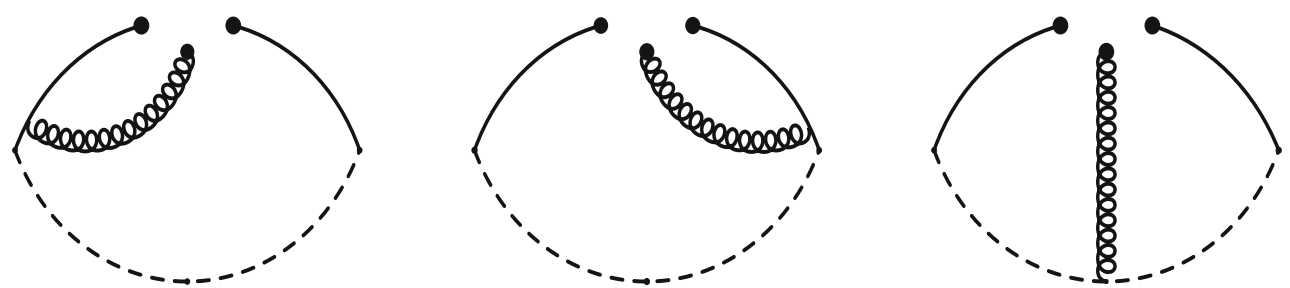

Fig. 3 The diagrams contribute to the mixed condensate $\left\langle\bar{q} g_{s} \sigma G q\right\rangle$
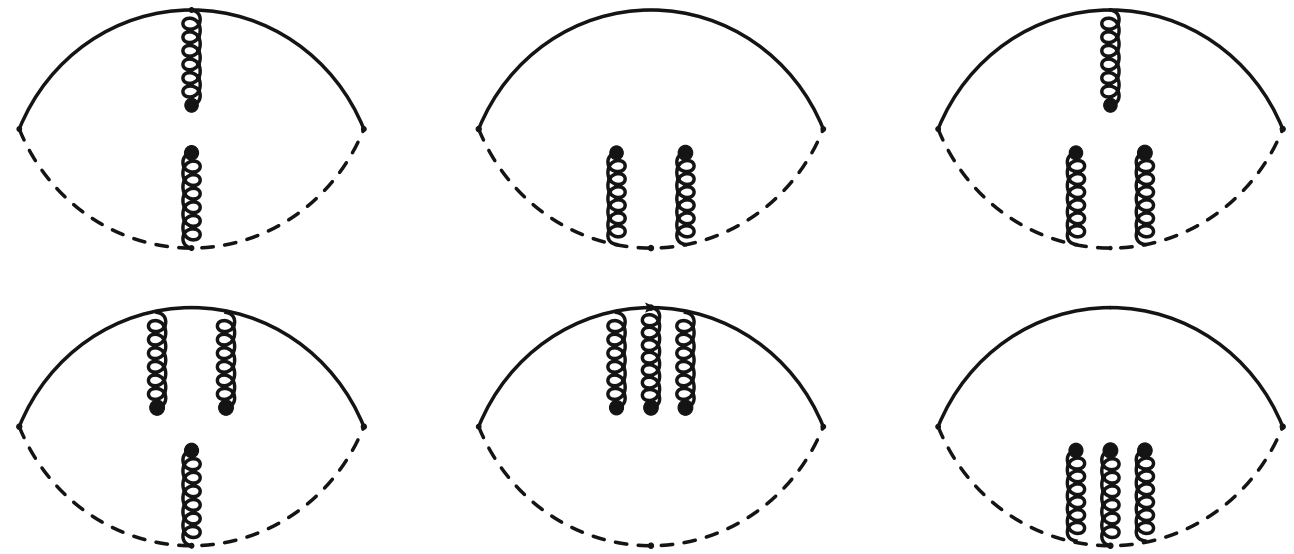

Fig. 4 The diagrams contribute to the gluon condensate $\left\langle\frac{\alpha_{s} G G}{\pi}\right\rangle$ and three-gluon condensate $\left\langle g_{s}^{3} G G G\right\rangle$
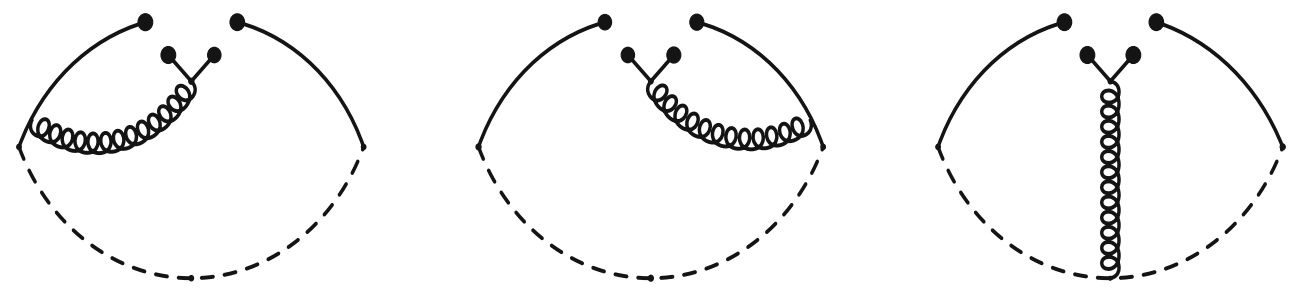

Fig. 5 The diagrams contribute to the four-quark condensate $\langle\bar{q} q\rangle^{2}$

$$
\begin{aligned}
& B_{T} \Pi_{V}=B_{T} \Pi_{V}^{0}+B_{T} \Pi_{V}^{3}+B_{T} \Pi_{V}^{4}+B_{T} \Pi_{V}^{5}+B_{T} \Pi_{V}^{6}, \\
& B_{T} \Pi_{A}=\left.B_{T} \Pi_{V}\right|_{m_{Q} \rightarrow-m_{Q}},
\end{aligned}
$$

$$
B_{T} \Pi_{5}^{0}=\frac{3}{8 \pi^{2}} \int_{m_{Q}^{2}}^{s_{0}} \mathrm{~d} s s\left(1-\frac{m_{Q}^{2}}{s}\right)^{2}\left\{1+\frac{2 m_{q} m_{Q}}{s-m_{Q}^{2}}\right.
$$




$$
\begin{aligned}
& \left.+\frac{4 \alpha_{s}}{3 \pi} R_{5}\left(\frac{m_{Q}^{2}}{s}\right)\right\} \exp \left(-\frac{s}{T^{2}}\right) \\
B_{T} \Pi_{5}^{3}= & -m_{Q}\langle\bar{q} q\rangle\left\{1+\frac{\alpha_{s}}{\pi}\left[6-\frac{4 m_{Q}^{2}}{3 T^{2}}-\frac{2}{3}\left(1-\frac{m_{Q}^{2}}{T^{2}}\right)\right.\right. \\
& \left.\left.\times \log \frac{m_{Q}^{2}}{\mu^{2}}-2 \Gamma\left(0, \frac{m_{Q}^{2}}{T^{2}}\right) \exp \left(\frac{m_{Q}^{2}}{T^{2}}\right)\right]\right\} \\
& \times \exp \left(-\frac{m_{Q}^{2}}{T^{2}}\right)+\frac{m_{q}\langle\bar{q} q\rangle}{2}\left(1+\frac{m_{Q}^{2}}{T^{2}}\right) \\
& \times \exp \left(-\frac{m_{Q}^{2}}{T^{2}}\right), \\
B_{T} \Pi_{5}^{4}= & \frac{1}{12}\left\langle\frac{\alpha_{s} G G}{\pi}\right\rangle \exp \left(-\frac{m_{Q}^{2}}{T^{2}}\right) \\
B_{T} \Pi_{5}^{5}= & -\left\{\frac{m_{Q}\left\langle\bar{q} g_{s} \sigma G q\right\rangle}{2 T^{2}}\left(1-\frac{m_{Q}^{2}}{2 T^{2}}\right)\right. \\
& \left.+\frac{m_{q} m_{Q}^{4}\left\langle\bar{q} g_{s} \sigma G q\right\rangle}{12 T^{6}}\right\} \exp \left(-\frac{m_{Q}^{2}}{T^{2}}\right) \\
B_{T} \Pi_{5}^{6}= & -\frac{16 \pi \alpha_{s}\langle\bar{q} q\rangle^{2}}{27 T^{2}}\left(1+\frac{m_{Q}^{2}}{2 T^{2}}-\frac{m_{Q}^{4}}{12 T^{4}}\right) \exp \left(-\frac{m_{Q}^{2}}{T^{2}}\right) \\
& +\frac{\left\langle g_{s}^{3} G G G\right\rangle}{\pi^{2}}\left\{\frac{5}{192 T^{2}}+\frac{1}{768 m_{Q}^{2}}+\frac{5 m_{Q}^{2}}{1536 T^{4}}\right. \\
& \left.-\frac{m_{Q}^{4}}{768 T^{6}}-\left(\frac{m_{Q}^{2}}{128 T^{4}}+\frac{m_{Q}^{4}}{384 T^{6}}\right) \log \frac{m_{Q}^{2} \mu^{2}}{T^{4}}\right\} \\
& \times \exp \left(-\frac{m_{Q}^{2}}{T^{2}}\right),
\end{aligned}
$$

$$
\begin{aligned}
B_{T} \Pi_{V}^{0}= & \frac{1}{8 \pi^{2}} \int_{m_{Q}^{2}}^{s_{0}} \mathrm{~d} s s\left(1-\frac{m_{Q}^{2}}{s}\right)^{2}\left(2+\frac{m_{Q}^{2}}{s}\right) \\
& \times\left\{1+\frac{6 s m_{q} m_{Q}}{\left(s-m_{Q}^{2}\right)\left(2 s+m_{Q}^{2}\right)}+\frac{4 \alpha_{s}}{3 \pi} R_{V}\left(\frac{m_{Q}^{2}}{s}\right)\right\} \\
& \times \exp \left(-\frac{s}{T^{2}}\right)
\end{aligned}
$$

$$
B_{T} \Pi_{V}^{3}=-m_{Q}\langle\bar{q} q\rangle\left\{1+\frac{\alpha_{s}}{\pi}\left[\frac{8}{3}-\frac{4 m_{Q}^{2}}{3 T^{2}}+\frac{2}{3}\left(2+\frac{m_{Q}^{2}}{T^{2}}\right)\right.\right.
$$$$
\left.\left.\times \log \frac{m_{Q}^{2}}{\mu^{2}}-\frac{2 m_{Q}^{2}}{3 T^{2}} \Gamma\left(0, \frac{m_{Q}^{2}}{T^{2}}\right) \exp \left(\frac{m_{Q}^{2}}{T^{2}}\right)\right]\right\}
$$$$
\times \exp \left(-\frac{m_{Q}^{2}}{T^{2}}\right)+\frac{m_{q} m_{Q}^{2}\langle\bar{q} q\rangle}{2 T^{2}} \exp \left(-\frac{m_{Q}^{2}}{T^{2}}\right),
$$

$B_{T} \Pi_{V}^{4}=-\frac{1}{12}\left\langle\frac{\alpha_{s} G G}{\pi}\right\rangle \exp \left(-\frac{m_{Q}^{2}}{T^{2}}\right)$,

$B_{T} \Pi_{V}^{5}=\left\{\frac{m_{Q}^{3}\left\langle\bar{q} g_{s} \sigma G q\right\rangle}{4 T^{4}}+\frac{m_{q}\left\langle\bar{q} g_{s} \sigma G q\right\rangle}{12 T^{2}}\right.$

$$
\begin{aligned}
& \left.\times\left(1+\frac{m_{Q}^{2}}{T^{2}}-\frac{m_{Q}^{4}}{T^{4}}\right)\right\} \exp \left(-\frac{m_{Q}^{2}}{T^{2}}\right) \\
B_{T} \Pi_{V}^{6}= & -\frac{20 \pi \alpha_{s}\langle\bar{q} q\rangle^{2}}{81 T^{2}}\left(1+\frac{m_{Q}^{2}}{T^{2}}-\frac{m_{Q}^{4}}{5 T^{4}}\right) \exp \left(-\frac{m_{Q}^{2}}{T^{2}}\right) \\
& +\frac{\left\langle g_{s}^{3} G G G\right\rangle}{\pi^{2}}\left\{-\frac{1}{1152 T^{2}}+\frac{1}{115 m_{Q}^{2}}\right. \\
& +\frac{m_{Q}^{2}}{768 T^{4}}\left(1-\frac{m_{Q}^{2}}{T^{2}}\right)+\frac{55 m_{Q}^{2}}{4608 T^{4}} \\
& \left.+\frac{1}{192 T^{2}}\left(1+\frac{m_{Q}^{2}}{T^{2}}-\frac{m_{Q}^{4}}{2 T^{4}}\right) \log \frac{m_{Q}^{2} \mu^{2}}{T^{4}}\right\} \\
& \times \exp \left(-\frac{m_{Q}^{2}}{T^{2}}\right), \\
R_{5}(x)= & \frac{9}{4}+2 \operatorname{Li}_{2}(x)+\log x \log (1-x)-\frac{3}{2} \log \frac{1-x}{x} \\
& -\log (1-x)+x \log \frac{1-x}{x}-\frac{x}{1-x} \log x \\
R_{V}(x)= & \frac{13}{4}+2 \operatorname{Li}_{2}(x)+\log x \log (1-x)-\frac{3}{2} \log \frac{1-x}{x} \\
& -\log (1-x)+x \log \frac{1-x}{x}-\frac{x}{1-x} \log x \\
& +\frac{(3+x)(1-x)}{2+x} \log \frac{1-x}{x}-\frac{2 x}{(2+x)(1-x)^{2}} \\
& \times \log x-\frac{5+2 x}{2+x}-\frac{2 x}{(2+x)(1-x)} \\
& -\int_{0}^{x} \mathrm{~d} t \frac{1}{t} \log (1-t), \\
\operatorname{Li}_{2}(x) & \mathrm{d} t \frac{1}{t+x} \mathrm{e}^{-t}, \\
&
\end{aligned}
$$

and the $s_{0}$ are the continuum threshold parameters. The perturbative $\mathcal{O}\left(\alpha_{s}\right)$ corrections $R_{5}(x)$ and $R_{V}(x)$ are taken from Refs. [27,28]. We can also take into account the semianalytical perturbative $\mathcal{O}\left(\alpha_{s}^{2}\right)$ corrections to the perturbative terms for $B_{T} \Pi_{5}^{0}$,

$$
\begin{gathered}
\frac{1}{8 \pi^{2}}\left(\frac{\alpha_{s}}{\pi}\right)^{2} \int_{m_{c}^{2}}^{s_{0}} \mathrm{~d} s\left\{\frac{16}{9} \mathrm{R} 2 \mathrm{sFF}[v]+4 \mathrm{R} 2 \mathrm{sFA}[v]\right. \\
\left.+\frac{2 n_{l}}{3} \mathrm{R} 2 \mathrm{sFL}[v]+\frac{2}{3} \mathrm{R} 2 \mathrm{sFH}[v]\right\} \exp \left(-\frac{s}{T^{2}}\right),
\end{gathered}
$$

where $\mathrm{R} 2 \mathrm{sFF}[v], \mathrm{R} 2 \mathrm{sFA}[v], \mathrm{R} 2 \mathrm{sFL}[v]$, and $\mathrm{R} 2 \mathrm{sFH}[v]$ with the variable $v=\left(1-\frac{m_{c}^{2}}{s}\right) /\left(1+\frac{m_{c}^{2}}{s}\right)$ are mathematical functions defined at the energy scale of the pole mass $\mu=m_{c} ; n_{l}$ counts the number of massless quarks $[29,30]$.

We can derive Eqs. (9)-(12) with respect to $1 / T^{2}$, and then eliminate the decay constants $f_{S / P / V / A}$ to obtain the QCD sum rules for the masses.

$m_{S / P / V / A}^{2}=\frac{-\frac{\mathrm{d}}{\mathrm{d}\left(1 / T^{2}\right)} B_{T} \Pi_{0 / 5 / V / A}}{B_{T} \Pi_{0 / 5 / V / A}}$. 
Once the masses $m_{S / P / V / A}$ are obtained, we can take them as input parameters and obtain the decay constants from the QCD sum rules in Eqs. (9)-(12).

In the case of the light-quark currents, the perturbative $\mathcal{O}\left(\alpha_{s}\right)$ corrections to the perturbative terms amount to multiplying the factors $1+\frac{11}{3} \frac{\alpha_{s}}{\pi} \approx 1+3.67 \frac{\alpha_{s}}{\pi}$ and $1+\frac{\alpha_{s}}{\pi}$ to the perturbative terms in the correlation functions for the pseudoscalar (scalar) and vector (axial-vector) currents, respectively [53]. In the present case, if we take the approximation $\mu^{2}=m_{c}^{2}=T^{2}$, the perturbative $\mathcal{O}\left(\alpha_{s}\right)$ corrections to the quark condensate terms amount to multiplying the factors $1+3.47 \frac{\alpha_{s}}{\pi}$ and $1+0.94 \frac{\alpha_{s}}{\pi}$ to the quark condensate terms in the correlation functions for the pseudoscalar (scalar) and vector (axial-vector) currents, respectively. The analogous $\mathcal{O}\left(\alpha_{s}\right)$ corrections indicate that the present calculations are reliable.

\section{Numerical results and discussions}

In the heavy quark limit, the heavy-light mesons $Q \bar{q}$ can be classified in doublets according to the total angular momentum of the light antiquark $\vec{s}_{\ell}, \vec{s}_{\ell}=\vec{s}_{\bar{q}}+\vec{L}$, where $\vec{s}_{\bar{q}}$ and $\vec{L}$ are the spin and orbital angular momentum of the light antiquark, respectively. The spin doublets $\left(D, D^{*}\right),\left(D_{s}, D_{s}^{*}\right)$, ( $\left.D_{0}^{*}(2400), D_{1}(2430)\right),\left(D_{s 0}^{*}(2317), D_{s 1}(2460)\right),\left(B, B^{*}\right)$, $\left(B_{s}, B_{s}^{*}\right)$ have been observed, the masses are $m_{D^{ \pm}}=$ $(1869.5 \pm 0.4) \mathrm{MeV}, m_{D^{0}}=(1864.84 \pm 0.07) \mathrm{MeV}$, $m_{D^{*}(2010)^{ \pm}}=(2010.26 \pm 0.07) \mathrm{MeV}, m_{D^{*}(2007)^{0}}=$ $(2006.96 \pm 0.10) \mathrm{MeV}, m_{D_{0}^{*}(2400)^{0}}=(2318 \pm 29) \mathrm{MeV}$, $m_{D_{0}^{*}(2400)^{ \pm}}=(2403 \pm 14 \pm 35) \mathrm{MeV}, m_{D_{1}(2430)^{0}}=(2427 \pm$ $26 \pm 25) \mathrm{MeV}, m_{D_{s}^{ \pm}}=(1969.0 \pm 1.4) \mathrm{MeV}, m_{D_{s}^{*}(2112)^{ \pm}}=$ $(2112.1 \pm 0.4) \mathrm{MeV}, m_{D_{s 0}^{*}(2317)^{ \pm}}=(2318.0 \pm 1.0) \mathrm{MeV}$, $m_{D_{s 1}(2460)^{ \pm}}=(2459.6 \pm 0.9) \mathrm{MeV}, m_{B^{ \pm}}=(5279.25 \pm$ $0.26) \mathrm{MeV}, m_{B^{0}}=(5279.55 \pm 0.26) \mathrm{MeV}, m_{B^{*}}=(5325.2$ $\pm 0.4) \mathrm{MeV}, m_{B_{s}}=(5366.7 \pm 0.4) \mathrm{MeV}, m_{B_{s}^{*}}=(5415.8 \pm$ 1.5) $\mathrm{MeV}$ from the Particle Data Group [1]. The spin doublets $\left(B_{0}^{*}, B_{1}\right)$ and $\left(B_{s 0}^{*}, B_{s 1}\right)$ have not been observed yet. The doublet $(D(2550), D(2600))$ or $\left(D_{J}(2580), D_{J}^{*}(2650)\right)$ is tentatively identified as the first radial excited state of the doublet $\left(D, D^{*}\right)$, the doublet $\left(?, D_{s 1}^{*}(2700)\right)$ is tentatively identified as the first radial excited state of the doublet $\left(D_{s}, D_{s}^{*}(2112)\right)$ [55-57].

We take the values $\sqrt{s_{0}}=m_{\mathrm{gr}}+(0.4-0.8) \mathrm{GeV}$ as guides, here gr denotes the ground states, and we search for the optimal threshold parameters $s_{0}$ and Borel parameters $T^{2}$ to satisfy the following criteria:

- pole dominance at the phenomenological side;

- convergence of the operator product expansion;

- appearance of the Borel platforms;

- reappearance of experimental values of the ground state heavy meson masses.
The contributions of the ground states can be fully taken into account by choosing the threshold parameters $\sqrt{s_{0}}=$ $m_{\text {gr }}+(0.4-0.8) \mathrm{GeV}$. The contaminations of the excited states are very small if there are some contaminations, we expect that the couplings of the currents to the excited states are more weak than that to the ground states. For example, the decay constants of the pseudoscalar mesons $\pi(140)$ and $\pi(1800)$ have the hierarchy $f_{\pi(1300)} \ll f_{\pi(140)}$ from the lattice QCD [58], the QCD sum rules [59], or from the experimental data [60].

The vacuum condensates are taken to be the standard values $\langle\bar{u} u\rangle=\langle\bar{d} d\rangle=-(0.24 \pm 0.01 \mathrm{GeV})^{3},\langle\bar{s} s\rangle=(0.8 \pm$ $0.1)\langle\bar{u} u\rangle,\left\langle\bar{q} g_{s} \sigma G q\right\rangle=m_{0}^{2}\langle\bar{q} q\rangle, m_{0}^{2}=(0.8 \pm 0.1) \mathrm{GeV}^{2}$, $\left\langle\frac{\alpha_{s} G G}{\pi}\right\rangle=(0.33 \mathrm{GeV})^{4},\left\langle g_{s}^{3} G G G\right\rangle=0.045 \mathrm{GeV}^{6}$ at the energy scale $\mu=1 \mathrm{GeV}[52,53]$. The quark condensates and mixed quark condensates evolve with the renormalization group equation, $\langle\bar{q} q\rangle(\mu)=\langle\bar{q} q\rangle(Q)\left[\frac{\alpha_{s}(Q)}{\alpha_{s}(\mu)}\right]^{\frac{4}{9}}$ and $\left\langle\bar{q} g_{s} \sigma G q\right\rangle(\mu)=\left\langle\bar{q} g_{s} \sigma G q\right\rangle(Q)\left[\frac{\alpha_{s}(Q)}{\alpha_{s}(\mu)}\right]^{\frac{2}{27}}$.

In the article, we take the $\overline{M S}$ masses $m_{b}\left(m_{b}\right)=(4.18 \pm$ $0.03) \mathrm{GeV}, m_{c}\left(m_{c}\right)=(1.275 \pm 0.025) \mathrm{GeV}$, and $m_{s}(\mu=$ $2 \mathrm{GeV})=(0.095 \pm 0.005) \mathrm{GeV}$ from the Particle Data Group [1], and we take into account the energy-scale dependence of the $\overline{M S}$ masses from the renormalization group equation,

$$
\begin{aligned}
m_{b}(\mu)= & m_{b}\left(m_{b}\right)\left[\frac{\alpha_{s}(\mu)}{\alpha_{s}\left(m_{b}\right)}\right]^{\frac{12}{23}}, \\
m_{c}(\mu)= & m_{c}\left(m_{c}\right)\left[\frac{\alpha_{s}(\mu)}{\alpha_{s}\left(m_{c}\right)}\right]^{\frac{12}{25}}, \\
m_{s}(\mu)= & m_{s}(2 \mathrm{GeV})\left[\frac{\alpha_{s}(\mu)}{\alpha_{s}(2 \mathrm{GeV})}\right]^{\frac{4}{9}}, \\
m_{u / d}(\mu)= & m_{u / d}(1 \mathrm{GeV})\left[\frac{\alpha_{s}(\mu)}{\alpha_{s}(1 \mathrm{GeV})}\right]^{\frac{4}{9}}, \\
\alpha_{s}(\mu)= & \frac{1}{b_{0} t}\left[1-\frac{b_{1}}{b_{0}^{2}} \frac{\log t}{t}\right. \\
& \left.+\frac{b_{1}^{2}\left(\log ^{2} t-\log t-1\right)+b_{0} b_{2}}{b_{0}^{4} t^{2}}\right],
\end{aligned}
$$

where $t=\log \frac{\mu^{2}}{\Lambda^{2}}, b_{0}=\frac{33-2 n_{f}}{12 \pi}, b_{1}=\frac{153-19 n_{f}}{24 \pi^{2}}, b_{2}=$ $\frac{2857-\frac{5033}{9} n_{f}+\frac{325}{27} n_{f}^{2}}{128 \pi^{3}}, \Lambda=213,296$, and $339 \mathrm{MeV}$ for the flavors $n_{f}=5,4$, and 3, respectively [1]. Furthermore, we obtain the values $m_{u}=m_{d}=6 \mathrm{MeV}$ from the Gell-MannOakes-Renner relation at the energy scale $\mu=1 \mathrm{GeV}$.

In this article, we choose the $\overline{M S}$ masses by setting $m=$ $m(\mu)$ and take the perturbative $\mathcal{O}\left(\alpha_{S}\right)$ corrections to the perturbative terms. In other words, we take the $R_{5 / V}\left(\frac{m_{Q}^{2}}{s}\right)$ only. In calculations, we take $n_{f}=3$ and $\mu_{D / D^{*}}=\sqrt{m_{D}^{2}-m_{c}^{2}} \approx$ 
Table 1 The Borel parameters, continuum threshold parameters, pole contributions, masses, and decay constants of the heavy-light mesons

\begin{tabular}{llllll}
\hline & $T^{2}\left(\mathrm{GeV}^{2}\right)$ & $s_{0}\left(\mathrm{GeV}^{2}\right)$ & Pole $(\%)$ & $m_{S / P / V / A}(\mathrm{GeV})$ & $f_{S / P / V / A}(\mathrm{MeV})$ \\
\hline$D$ & $1.2-1.8$ & $6.2 \pm 0.5$ & $(67-93)$ & $1.87 \pm 0.10$ & $208 \pm 10$ \\
$D_{s}$ & $1.3-1.9$ & $7.3 \pm 0.5$ & $(77-96)$ & $1.97 \pm 0.10$ & $240 \pm 10$ \\
$D^{*}$ & $1.9-2.5$ & $6.4 \pm 0.5$ & $(51-76)$ & $2.01 \pm 0.08$ & $263 \pm 21$ \\
$D_{s}^{*}$ & $1.8-2.4$ & $7.5 \pm 0.5$ & $(66-87)$ & $2.11 \pm 0.07$ & $308 \pm 21$ \\
$D_{0}^{*}$ & $2.4-3.0$ & $8.3 \pm 0.5$ & $(59-78)$ & $2.40 \pm 0.05$ & $373 \pm 19$ \\
$D_{s 0}^{*}$ & $2.1-2.7$ & $7.4 \pm 0.5$ & $(55-77)$ & $2.32 \pm 0.05$ & $333 \pm 20$ \\
$D_{1}$ & $2.9-3.5$ & $8.6 \pm 0.5$ & $(52-70)$ & $2.42 \pm 0.05$ & $332 \pm 18$ \\
$D_{s 1}$ & $2.7-3.3$ & $9.3 \pm 0.5$ & $(61-78)$ & $2.46 \pm 0.06$ & $245 \pm 17$ \\
$B$ & $5.5-6.5$ & $34.0 \pm 1.0$ & $(44-63)$ & $5.28 \pm 0.07$ & $194 \pm 15$ \\
$B_{s}$ & $5.3-6.3$ & $36.0 \pm 1.0$ & $(56-74)$ & $5.37 \pm 0.07$ & $231 \pm 16$ \\
$B^{*}$ & $6.0-7.0$ & $34.5 \pm 1.0$ & $(45-62)$ & $5.32 \pm 0.06$ & $213 \pm 18$ \\
$B_{s}^{*}$ & $6.1-7.1$ & $36.5 \pm 1.0$ & $(53-69)$ & $5.42 \pm 0.06$ & $255 \pm 19$ \\
$B_{0}^{*}$ & $6.3-7.3$ & $40.0 \pm 1.0$ & $(60-75)$ & $5.72 \pm 0.05$ & $281 \pm 14$ \\
$B_{s 0}^{*}$ & $6.5-7.5$ & $40.0 \pm 1.0$ & $(60-74)$ & $5.70 \pm 0.06$ & $274 \pm 13$ \\
$B_{1}$ & $6.7-7.7$ & $41.0 \pm 1.0$ & $(62-75)$ & $5.74 \pm 0.05$ & $335 \pm 18$ \\
$B_{s 1}$ & $7.1-8.1$ & $42.0 \pm 1.0$ & $(63-76)$ & $5.76 \pm 0.06$ & $348 \pm 18$ \\
\hline
\end{tabular}

$1 \mathrm{GeV}$ for the S-wave mesons $D$ and $D^{*} ; n_{f}=4$ and $\mu_{B / B^{*}}=\sqrt{m_{B}^{2}-m_{b}^{2}} \approx 2.5 \mathrm{GeV}$ for the $\mathrm{S}$-wave mesons $B$ and $B^{*}$. If we count the contribution of the additional P-wave as $0.5 \mathrm{GeV}$, then $\mu_{D_{0}^{*} / D_{1}}=1.5 \mathrm{GeV}$ and $\mu_{B_{0}^{*} / B_{1}}=3.0 \mathrm{GeV}$. On the other hand, we take into account the $S U$ (3) breaking effect, which is supposed to be $100 \mathrm{MeV}$ for the light quarks, then $\mu_{D_{s} / D_{s}^{*}}=1.1 \mathrm{GeV}, \mu_{B_{s} / B_{s}^{*}}=2.6 \mathrm{GeV}, \mu_{D_{s 0}^{*} / D_{s 1}}=$ $1.6 \mathrm{GeV}$, and $\mu_{B_{s 0}}^{*} / B_{s 1}=3.1 \mathrm{GeV}$. Those energy scales work well.

The continuum threshold parameters, Borel parameters, pole contributions are shown explicitly in Table 1. From Table 1, we can see that the pole dominance can be satisfied. On the other hand, the dominant contributions come from the perturbative terms and the quark condensate terms, so we expect to obtain reliable predictions.

After taking into account the uncertainties of the input parameters, we obtain the values of the masses and decay constants of the heavy-light mesons, which are shown in Figs. 6, 7, 8 and 9 and Table 1. From the figures, we can see that the masses and decays constants are rather stable with variations of the Borel parameters $T^{2}$, the predictions are reasonable.

From Table 1, we can see that the experimental values of the masses of the observed mesons $\left(D, D^{*}\right),\left(D_{s}, D_{s}^{*}\right)$, ( $\left.D_{0}^{*}(2400), D_{1}(2430)\right),\left(D_{s 0}^{*}(2317), D_{s 1}(2460)\right),\left(B, B^{*}\right)$, $\left(B_{s}, B_{s}^{*}\right)$ can be well reproduced. The masses of the $\left(B_{0}^{*}, B_{1}\right)$, and $\left(B_{s 0}^{*}, B_{s 1}\right)$ vary in rather large ranges from different theoretical approaches, $m_{B_{0}^{*}}=(5.53-5.76) \mathrm{GeV}, m_{B_{1}}=$ $(5.58-5.78) \mathrm{GeV}, m_{B_{s 0}^{*}}=(5.63-5.83) \mathrm{GeV}, m_{B_{s 1}}=$ (5.67-5.86) GeV, for a comprehensive review, one can consult Ref. [61]. The present predictions $m_{B_{0}^{*}}=(5.72 \pm$ $0.05) \mathrm{GeV}, m_{B_{1}}=(5.74 \pm 0.05) \mathrm{GeV}, m_{B_{s 0}^{*}}=(5.70 \pm$
$0.06) \mathrm{GeV}, m_{B_{s 1}}=(5.76 \pm 0.06) \mathrm{GeV}$ are compatible with those values.

The thresholds of the $D K, D^{*} K, B K$, and $B^{*} K$ states are $m_{D K}=2.36 \mathrm{GeV}, m_{D^{*} K}=2.50 \mathrm{GeV}, m_{B K}=5.78 \mathrm{GeV}$, and $m_{B^{*} K}=5.82 \mathrm{GeV}$, respectively. The $D_{s 0}^{*}$ (2317) and $D_{s 1}$ (2460) lie below the thresholds $m_{D K}$ and $m_{D^{*} K}$, respectively, the Okubo-Zweig-Iizuka allowed strong decays $D_{s 0}^{*}(2317) \rightarrow D K$ and $D_{s 1}(2460) \rightarrow D^{*} K$ are kinematically forbidden, the widths of $D_{s 0}^{*}(2317)$ and $D_{s 1}(2460)$ are very narrow. According to the present predictions $m_{B_{s 0}^{*}}=$ $(5.70 \pm 0.06) \mathrm{GeV}$ and $m_{B_{s 1}}=(5.76 \pm 0.06) \mathrm{GeV}$, the $B_{s 0}^{*}$ and $B_{s 1}$ also lie below the corresponding $B K$ and $B^{*} K$ thresholds, respectively. The strong decays $B_{s 0}^{*} \rightarrow B K$ and $B_{s 1} \rightarrow B^{*} K$ are kinematically forbidden, the P-wave heavy mesons $B_{s 0}^{*}$ and $B_{s 1}$ can decay through the isospin violation precesses $B_{s 0}^{*} \rightarrow B_{s} \eta \rightarrow B_{s} \pi^{0}$ and $B_{s 1} \rightarrow B_{s}^{*} \eta \rightarrow B_{s}^{*} \pi^{0}$, respectively, or through the radiative decays [62-64]. The $\eta$ and $\pi^{0}$ transition matrix is very small according to Dashen's theorem [65], $t_{\eta \pi}=\left\langle\pi^{0}|\mathcal{H}| \eta\right\rangle=-0.003 \mathrm{GeV}^{2}$, the P-wave bottomed mesons $B_{s 0}^{*}$ and $B_{s 1}$, just like their charmed cousins $D_{s 0}^{*}(2317)$ and $D_{s 1}(2460)$, maybe very narrow [66-68]. The present predictions are consistent with our previous work [22], but the analysis is refined by including more terms in the operator product expansion.

The values of the decay constants of the pseudoscalar mesons are slightly different from the ones in our previous work [13]. In Table 2, we compare the present predictions to the experimental data and other theoretical calculations, such the QCD sum rules (QCDSR) [2-7,23] and lattice QCD (LQCD) [8-11]. The present predictions $f_{D}=$ $(208 \pm 10) \mathrm{MeV}$ and $f_{B}=(194 \pm 15) \mathrm{MeV}$ are consistent with the experimental data within uncertainties, while the 

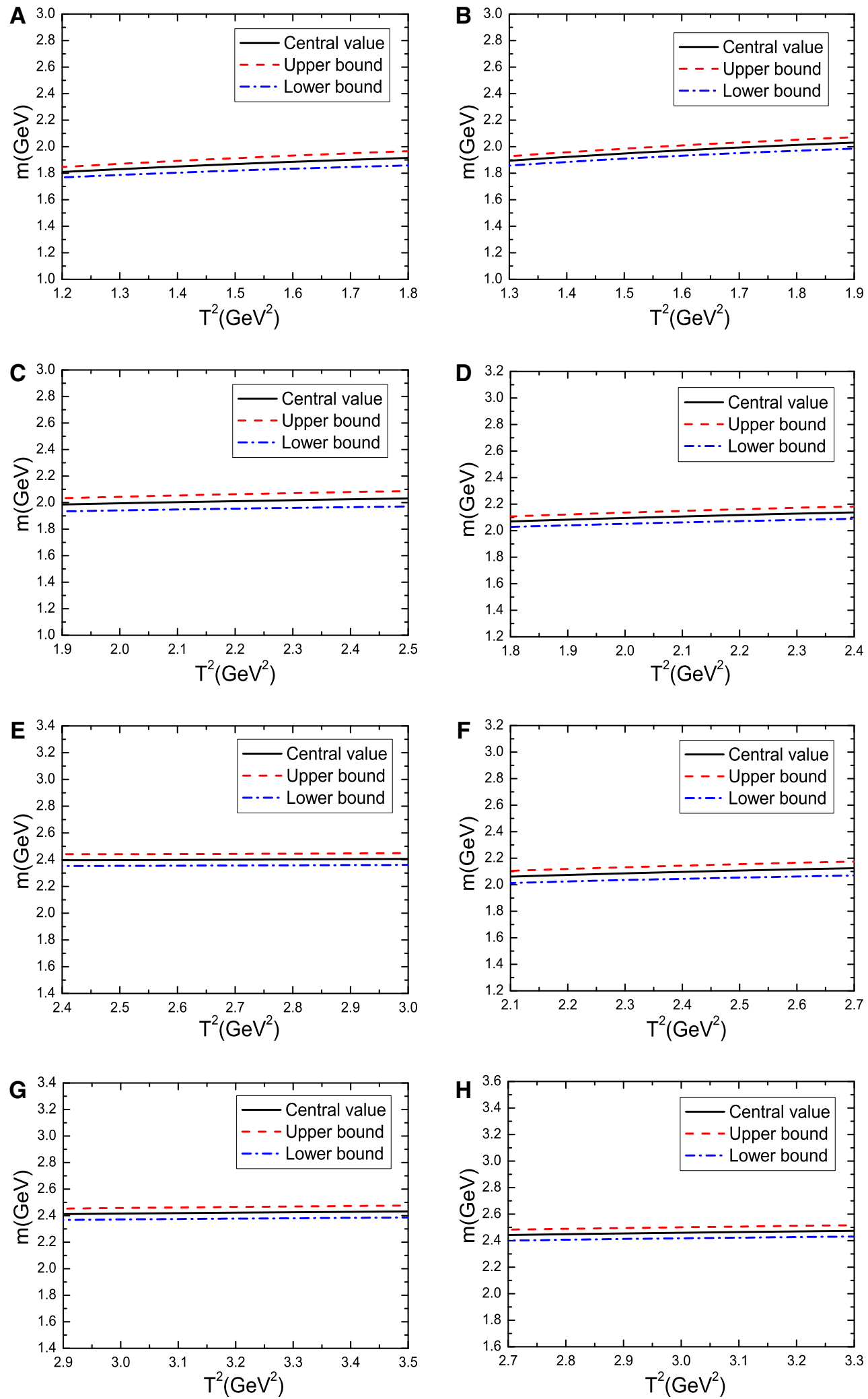

Fig. 6 The masses of the charmed mesons with variations of the Borel parameters $T^{2}, A, B, C, D, E, F, G$, and $H$ denote the mesons $D, D_{s}$, $D^{*}, D_{s}^{*}, D_{0}^{*}, D_{s 0}^{*}, D_{1}$, and $D_{s 1}$, respectively 

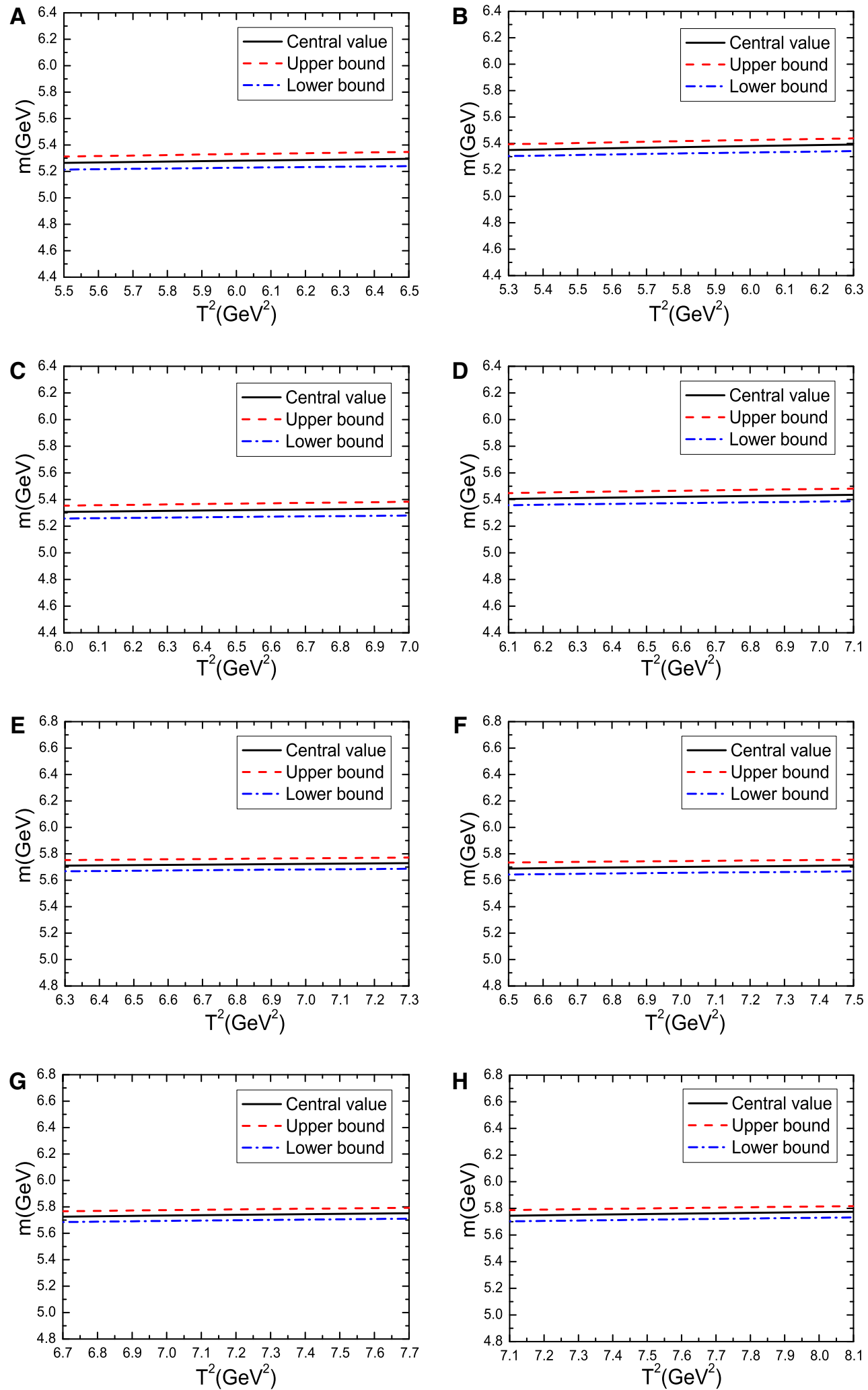

Fig. 7 The masses of the bottom mesons with variations of the Borel parameters $T^{2}, A, B, C, D, E, F, G$, and $H$ denote the mesons $B, B_{s}, B^{*}$, $B_{s}^{*}, B_{0}^{*}, B_{s 0}^{*}, B_{1}$, and $B_{s 1}$, respectively 

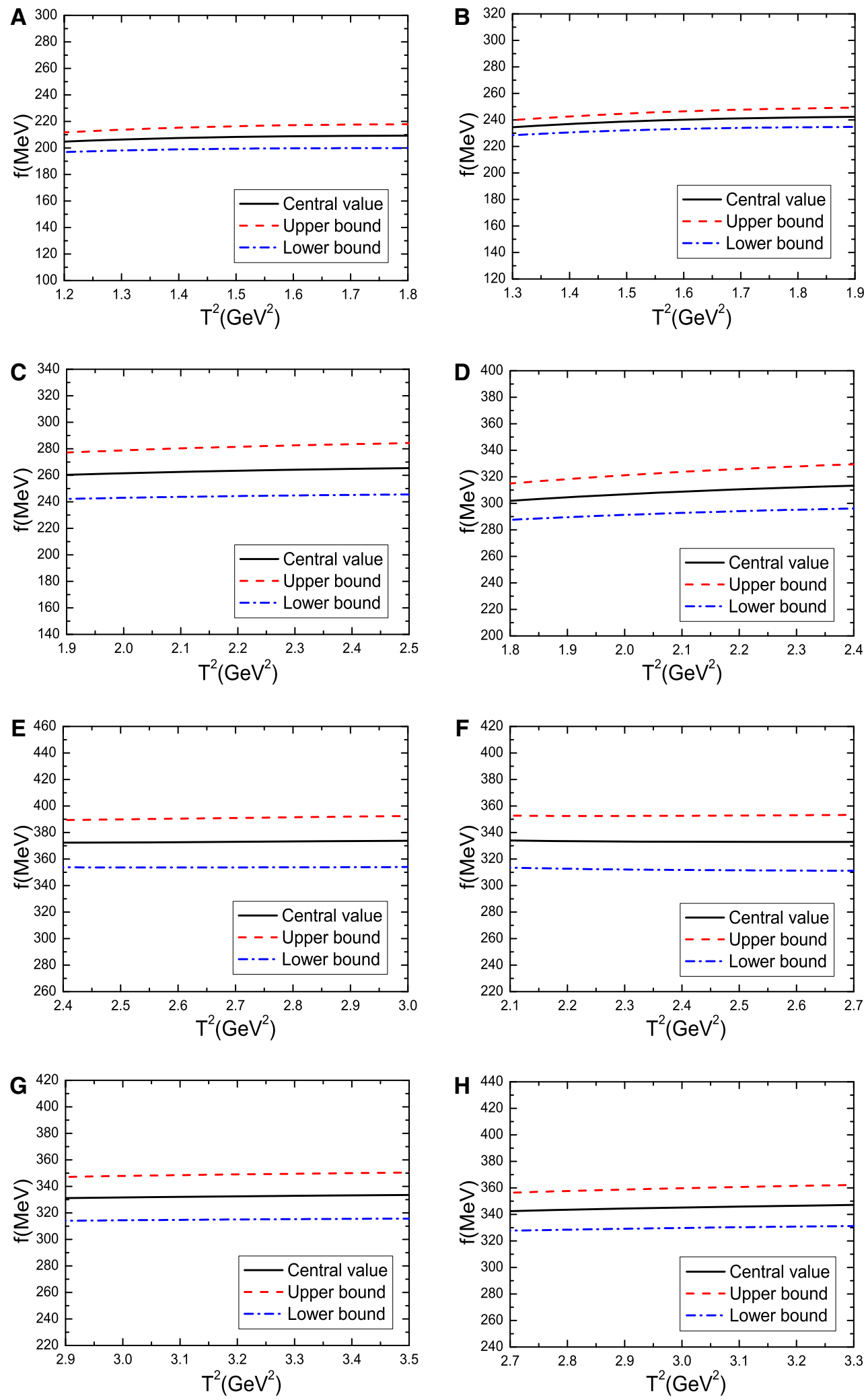

Fig. 8 The decay constants of the charmed mesons with variations of the Borel parameters $T^{2}, A, B, C, D, E, F, G$, and $H$ denote the mesons $D, D_{s}, D^{*}, D_{s}^{*}, D_{0}^{*}, D_{s 0}^{*}, D_{1}$, and $D_{s 1}$, respectively 

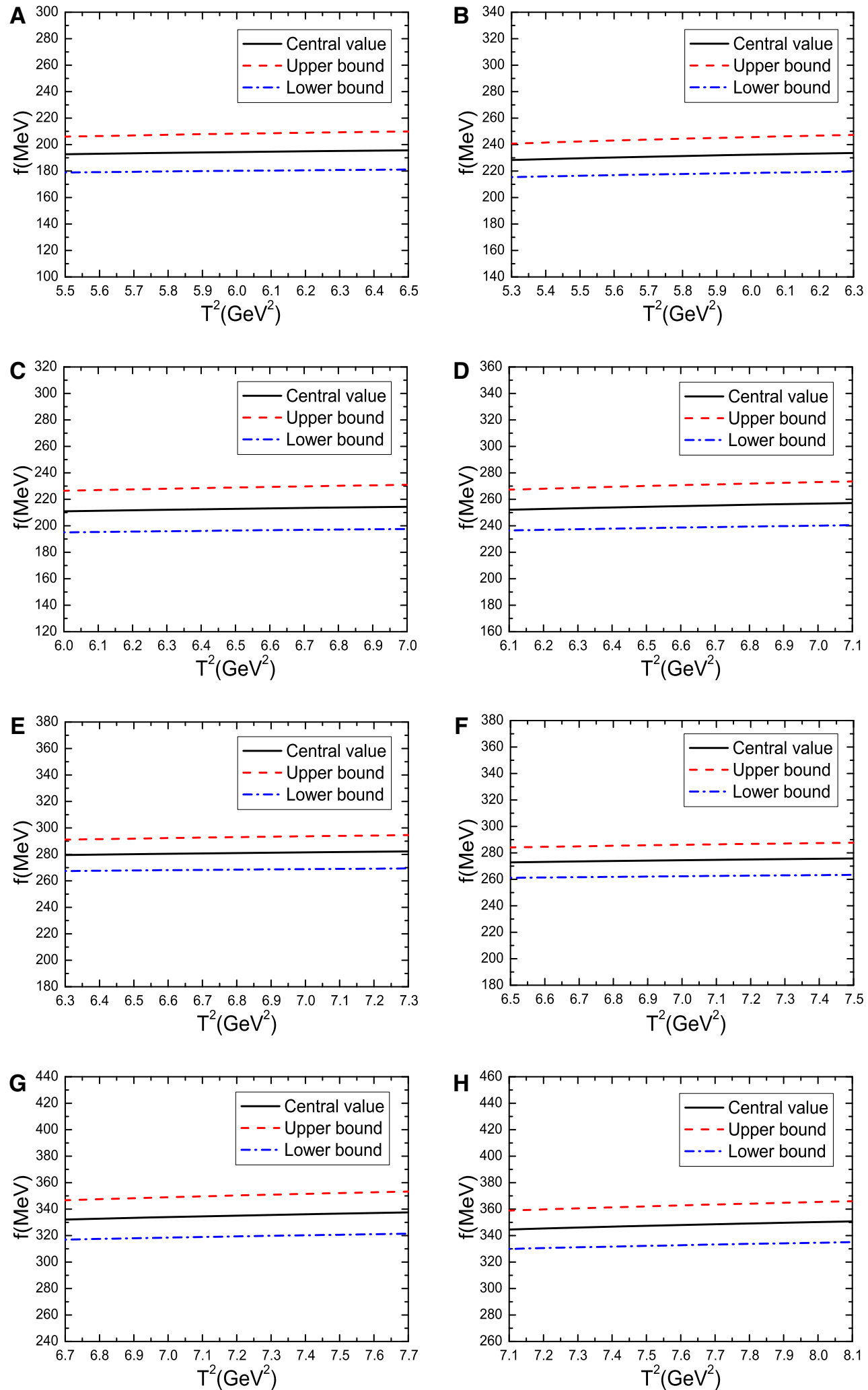

Fig. 9 The decay constants of the bottom mesons with variations of the Borel parameters $T^{2}, A, B, C, D, E, F$, $G$, and $H$ denote the mesons $B$, $B_{s}, B^{*}, B_{s}^{*}, B_{0}^{*}, B_{s 0}^{*}, B_{1}$, and $B_{s 1}$, respectively 
Table 2 The decay constants of the heavy pseudoscalar mesons from the experimental data, the QCD sum rules and lattice QCD, the superscript ${ }^{a}$ denotes that the pole masses are chosen and perturbative $\mathcal{O}\left(\alpha_{s}^{2}\right)$ corrections are taken into account

\begin{tabular}{|c|c|c|c|c|c|c|}
\hline & $f_{D}(\mathrm{MeV})$ & $f_{D_{s}}(\mathrm{MeV})$ & $f_{B}(\mathrm{MeV})$ & $f_{B_{s}}(\mathrm{MeV})$ & $f_{D_{s}} / f_{D}$ & $f_{B_{s}} / f_{B}$ \\
\hline Expt [1] & $204.6 \pm 5.0$ & $257.5 \pm 4.6$ & $190.6 \pm 4.7$ & & $1.258 \pm 0.038$ & \\
\hline QCDSR $[2,3]$ & $177 \pm 21$ & $205 \pm 22$ & $178 \pm 14$ & $200 \pm 14$ & $1.16 \pm 0.16$ & $1.12 \pm 0.11$ \\
\hline QCDSR [4] & $204 \pm 6$ & $246 \pm 6$ & $207 \pm 8$ & $234 \pm 5$ & $1.21 \pm 0.04$ & $1.14 \pm 0.03$ \\
\hline QCDSR [5,6] & $206.2 \pm 7.3$ & $245.3 \pm 15.7$ & $193.4 \pm 12.3$ & $232.5 \pm 18.6$ & $1.193 \pm 0.025$ & $1.203 \pm 0.020$ \\
\hline QCDSR [7] & $201_{-13}^{+12}$ & $238_{-23}^{+13}$ & $207_{-09}^{+17}$ & $242_{-12}^{+17}$ & $1.18_{-0.05}^{+0.04}$ & $1.17_{-0.04}^{+0.03}$ \\
\hline QCDSR [23] & & & $186 \pm 14$ & $222 \pm 12$ & & $1.19 \pm 0.09$ \\
\hline LQCD [8] & $197 \pm 9$ & $244 \pm 8$ & & & $1.24 \pm 0.03$ & \\
\hline LQCD $[9,10]$ & $213 \pm 4$ & $248.0 \pm 2.5$ & $191 \pm 9$ & $228 \pm 10$ & $1.164 \pm 0.018$ & $1.188 \pm 0.018$ \\
\hline LQCD [11] & $218.9 \pm 11.3$ & $260.1 \pm 10.8$ & $196.9 \pm 8.9$ & $242.0 \pm 9.5$ & $1.188 \pm 0.025$ & $1.229 \pm 0.026$ \\
\hline This work & $208 \pm 10$ & $240 \pm 10$ & $194 \pm 15$ & $231 \pm 16$ & $1.15 \pm 0.06$ & $1.19 \pm 0.10$ \\
\hline This work $^{\mathrm{a}}$ & $210 \pm 11$ & $259 \pm 10$ & $192 \pm 13$ & $230 \pm 13$ & $1.23 \pm 0.07$ & $1.20 \pm 0.09$ \\
\hline
\end{tabular}

prediction $f_{D_{s}}=(240 \pm 10) \mathrm{MeV}$ is lies below the lower bound of the experimental value $f_{D_{s}}=(257.5 \pm 4.6) \mathrm{MeV}$ [1]. We take the $\overline{M S}$ mass $m_{c}(\mu)$ and truncate the perturbative corrections to the order $\mathcal{O}\left(\alpha_{S}\right)$, the experimental values of $f_{D}, f_{D_{s}}$, and $f_{D_{s}} / f_{D}$ cannot be reproduced consistently by the QCD sum rules. The existence of a charged Higgs boson or any other charged object beyond the standard model would modify the decay rates, see Eq. (1), therefore modify the values of the decay constants, for example, the leptonic decay widths are modified in two-Higgs-doublet models $[69,70]$. If the predictions of $f_{D}, f_{D_{s}}$ and $f_{D_{s}} / f_{D}$ based on the QCD sum rules are close to the true values, new physics beyond the standard model are favored so as to smear the discrepancies between the theoretical calculations and experimental data.

The analytical expression of the perturbative $\mathcal{O}\left(\alpha_{S}\right)$ corrections $R_{5}\left(\frac{m_{Q}^{2}}{s}\right)$ is well known [27], while the semianalytical perturbative $\mathcal{O}\left(\alpha_{s}^{2}\right)$ corrections are presented as mathematical functions $\mathrm{R} 2 \mathrm{sFF}[v], \mathrm{R} 2 \mathrm{sFA}[v], \mathrm{R} 2 \mathrm{sFL}[v]$, and $\mathrm{R} 2 \mathrm{sFH}[v]$ with the variable $v=\left(1-\frac{m_{Q}^{2}}{s}\right) /\left(1+\frac{m_{Q}^{2}}{s}\right)$ at the energy scale of the heavy quark pole mass $\mu=m_{Q}$ $[29,30]$. The analytical expressions of the terms which contain logarithms, such as $\log \frac{\mu^{2}}{m_{Q}^{2}}, \log \frac{\mu^{2}}{s}$, cannot be recovered, it is unreasonable to take other energy scale besides $m_{Q}$. Now we choose the pole masses $m_{Q}$ and take into account the semi-analytical perturbative $\mathcal{O}\left(\alpha_{s}^{2}\right)$ corrections by setting $n_{f}=4$ and $\mu=m_{c}$ for the $D\left(D_{s}\right)$ meson and $n_{f}=5$ and $\mu=m_{b}$ for the $B\left(B_{s}\right)$ meson.

The on-shell quark propagators have no infrared divergences in perturbation theory, which provides a perturbative definition of the quark masses. The full quark propagators have no poles because the quarks are confined, so the pole masses cannot be defined outside of perturbation theory. Furthermore, the pole masses cannot be used to arbitrarily high
Table 3 The Borel parameters, continuum threshold parameters, pole contributions, masses, and decay constants of the heavy pseudoscalar mesons when the perturbative $\mathcal{O}\left(\alpha_{s}^{2}\right)$ corrections are taken into account

\begin{tabular}{llrlll}
\hline & $T^{2}\left(\mathrm{GeV}^{2}\right)$ & $s_{0}\left(\mathrm{GeV}^{2}\right)$ & Pole $(\%)$ & $m_{P}(\mathrm{GeV})$ & $f_{P}(\mathrm{MeV})$ \\
\hline$D$ & $1.4-2.0$ & $5.5 \pm 0.5$ & $(55-85)$ & $1.87 \pm 0.06$ & $210 \pm 11$ \\
$D_{s}$ & $1.0-1.6$ & $7.4 \pm 0.5$ & $(86-98)$ & $1.97 \pm 0.07$ & $259 \pm 10$ \\
$B$ & $4.1-4.9$ & $33.0 \pm 1.0$ & $(55-75)$ & $5.28 \pm 0.04$ & $192 \pm 13$ \\
$B_{s}$ & $4.4-5.2$ & $35.0 \pm 1.0$ & $(61-79)$ & $5.37 \pm 0.04$ & $230 \pm 13$ \\
\hline
\end{tabular}

accuracy because of nonperturbative infrared effects in QCD. We choose the pole masses just because the semi-analytical perturbative $\mathcal{O}\left(\alpha_{s}^{2}\right)$ corrections are calculated by taking the pole mass $m_{Q}$ and setting the energy scale to be $\mu=m_{Q}$ $[29,30]$. The contributions of the $u, d$ masses are tiny and can be neglected safely. In calculations, we set the pole masses $m_{u}=m_{d}=0, m_{s}=150 \mathrm{MeV}$, and observe that the masses of the heavy pseudoscalar mesons increase monotonously with increase of the pole masses, the values of the pole masses $m_{c}=1.44 \mathrm{GeV}$ and $m_{b}=4.67 \mathrm{GeV}$ can lead to satisfactory values by choosing reasonable Borel parameters and threshold parameters. Those pole masses are different from the $\overline{M S}$ masses, for example, $m_{c}(\mu=1 \mathrm{GeV})=1.39 \mathrm{GeV}, m_{b}(\mu=$ $1 \mathrm{GeV})=6.07 \mathrm{GeV}, m_{c}(\mu=2 \mathrm{GeV})=1.13 \mathrm{GeV}$, $m_{b}(\mu=2 \mathrm{GeV})=4.87 \mathrm{GeV}$ from Eq. (29). The pole masses are energy-scale independent, therefore the energyscale dependence of the QCD spectral densities originates only from the vacuum condensates.

The Borel parameters, continuum threshold parameters, pole contributions, and the resulting masses and decay constants of the heavy pseudoscalar mesons are shown in Table 3 , the values are slightly different from the ones in our previous work [13]. From Tables 1 and 3, we can see that the present predictions $f_{D}=(210 \pm 11) \mathrm{MeV}, f_{D_{s}}=(259 \pm 10) \mathrm{MeV}$, 
Table 4 The decay constants of the heavy vector mesons from the some theoretical calculations

\begin{tabular}{lllll}
\hline & $f_{D^{*}}(\mathrm{MeV})$ & $f_{D_{s}^{*}}(\mathrm{MeV})$ & $f_{B^{*}}(\mathrm{MeV})$ & $f_{B_{s}^{*}}(\mathrm{MeV})$ \\
\hline QCDSR [7] & $242_{-12}^{+20}$ & $293_{-14}^{+19}$ & $210_{-12}^{+10}$ & $251_{-16}^{+13}$ \\
QCDSR [24,25] & $252.2 \pm 22.3 \pm 4$ & $305.5 \pm 26.8 \pm 5$ & $181.8 \pm 13.1 \pm 4$ & $213.6 \pm 18.2 \pm 6$ \\
QCDSR [32] & $250 \pm 11$ & $270 \pm 19$ & $209 \pm 8$ & $220 \pm 9$ \\
LQCD [33] & $278 \pm 13 \pm 10$ & $311 \pm 9$ & & \\
LQCD [34] & & $274 \pm 6$ & & $213 \pm 7$ \\
LQCD [35] & & & $175 \pm 6$ & $229 \pm 20$ \\
LQCD [36] & $245 \pm 20$ & $272 \pm 16$ & $196 \pm 24$ & 251 \\
RPM [41] & 310 & 315 & 219 & $230 \pm 12$ \\
FCM [44] & $273 \pm 13$ & $307 \pm 18$ & $200 \pm 10$ & $229_{-31}^{+32}$ \\
LFQM [47] & $245_{-34}^{+35}$ & $272_{-38}^{+39}$ & $196_{-27}^{+28}$ & $255 \pm 19$ \\
This work & $263 \pm 21$ & $308 \pm 21$ & $213 \pm 18$ & \\
\hline
\end{tabular}

Table 5 The decay constants of the heavy scalar mesons from the some theoretical calculations

\begin{tabular}{lllll}
\hline & $f_{D_{0}^{*}}(\mathrm{MeV})$ & $f_{D_{s 0}^{*}}(\mathrm{MeV})$ & $f_{B_{0}^{*}}(\mathrm{MeV})$ & $f_{B_{s 0}^{*}}(\mathrm{MeV})$ \\
\hline QCDSR [26] & & $128 \pm 13$ & & \\
LQCD [37] & $360 \pm 90$ & $340 \pm 110$ & & \\
This work & $373 \pm 19$ & $333 \pm 20$ & $281 \pm 14$ & $274 \pm 13$ \\
\hline
\end{tabular}

and $f_{B}=(192 \pm 13) \mathrm{MeV}$ are in excellent agreement with the experimental data within uncertainties [1]. The ratio $f_{D_{s}} / f_{D}=1.23 \pm 0.07$ is also in excellent agreement with the experimental data $f_{D_{s}} / f_{D}=1.258 \pm 0.038$ [1], which indicates that the perturbative $\mathcal{O}\left(\alpha_{s}^{2}\right)$ corrections should be taken into account. However, the pole masses $m_{Q}$ and energy scales $\mu=m_{Q}$ have be chosen, as the semi-analytical expressions are obtained at such conditions. In this case, new physics beyond the standard model are not favored, as the agreement between the experimental data and present theoretical calculations is already excellent.

In Table 4, we compare the present predictions for the decay constants of the heavy vector mesons to other theoretical calculations, such as the QCD sum rules [7,24,25,32], lattice QCD [33-36], the relativistic potential model (RPM) [41], the field-correlator method (FCM) [44], and the lightfront quark model [47]. From the table, we can see that the predictions differ from each other in one way or the other. In Table 5, we compare the present predictions for the decay constants of the heavy scalar mesons to the ones from the QCD sum rules [26] and lattice QCD [37]. From the table, we can see that the predictions are consistent with the ones from lattice calculations but differ greatly from the ones from the QCD sum rules.

If we turn off the perturbative $\mathcal{O}\left(\alpha_{s}\right)$ corrections to the quark condensates and choose the same parameters, such as the $\overline{M S}$ masses, Borel parameters, and continuum threshold parameters, etc., the masses and decay constants undergo
Table 6 The shifts of the masses and decay constants of the heavy-light mesons when the perturbative $\mathcal{O}\left(\alpha_{s}\right)$ corrections to the quark condensates are turned off. We can re-choose the Borel windows to warrant the mass-shifts $\delta m_{S / P / V / A}=0$, the resulting shifts of the decay constants are shown in the bracket. The sign $+0(-0)$ denotes the value $0<\delta m<1 \mathrm{MeV}(-1 \mathrm{MeV}<\delta m<0)$

\begin{tabular}{lll}
\hline & $\delta m_{S / P / V / A}(\mathrm{MeV})$ & $\delta f_{S / P / V / A}(\mathrm{MeV})$ \\
\hline$D\left(0^{-}\right)$ & +42 & $-9(-11)$ \\
$D_{s}\left(0^{-}\right)$ & +34 & $-6(-8)$ \\
$D^{*}\left(1^{-}\right)$ & +22 & $-6(-8)$ \\
$D_{s}^{*}\left(1^{-}\right)$ & +12 & $-3(-5)$ \\
$D_{0}^{*}\left(0^{+}\right)$ & -43 & $+2(+6)$ \\
$D_{s 0}^{*}\left(0^{+}\right)$ & -44 & $+1(+6)$ \\
$D_{1}\left(1^{+}\right)$ & -5 & $+1(+3)$ \\
$D_{s 1}\left(1^{+}\right)$ & -2 & $+1(+2)$ \\
$B\left(0^{-}\right)$ & +2 & $-3(-4)$ \\
$B_{S}\left(0^{-}\right)$ & +1 & $-2(-3)$ \\
$B^{*}\left(1^{-}\right)$ & +0 & $-3(-3)$ \\
$B_{s}^{*}\left(1^{-}\right)$ & +0 & $-2(-2)$ \\
$B_{0}^{*}\left(0^{+}\right)$ & -2 & $+1(+3)$ \\
$B_{s 0}^{*}\left(0^{+}\right)$ & -1 & $+1(+3)$ \\
$B_{1}\left(1^{+}\right)$ & -0 & $+3(+3)$ \\
$B_{s 1}\left(1^{+}\right)$ & -0 & $+2(+2)$ \\
\hline
\end{tabular}

reduction or increment in a definite way according to the spin and parity; see Table 6 . From the table, we can see that the mass-shifts of the $D$-mesons with $J^{P}=0^{ \pm}$are larger than $40 \mathrm{MeV}$, while the shifts of the masses and decay constants of all the $B$-mesons are small and can be neglected. We can re-choose the Borel windows to warrant the massshifts $\delta m_{S / P / V / A}=0$, and account for the net effects by the shifts of the decay constants $\delta f_{S / P / V / A}$, which are shown the bracket in Table 6 . From the table, we can see that the largest shift of the decay constant $\delta f_{D}=-11 \mathrm{MeV}$, which exceeds the total uncertainty of the decay constant $\delta f_{D}= \pm 10 \mathrm{MeV}$ 
(see Table 1), the shifts of the decay constants of the $D$ mesons with $J^{P}=0^{ \pm}, 1^{-}$are larger than $5 \mathrm{MeV}$, while for other mesons, the shifts of the decay constants $|\delta f| \leq 4 \mathrm{MeV}$. All in all, we should take into account the perturbative $\mathcal{O}\left(\alpha_{s}\right)$ corrections to the quark condensates in a comprehensive study.

\section{Conclusion}

In this article, we calculate the contributions of the vacuum condensates up to dimension- 6 , in including the $\mathcal{O}\left(\alpha_{S}\right)$ corrections to the quark condensates, in the operator product expansion. Then we study the masses and decay constants of the pseudoscalar, scalar, vector, and axial-vector heavy-light mesons with the QCD sum rules in a systematic way. In calculations, we take the $\overline{M S}$ masses and take into account the perturbative $\mathcal{O}\left(\alpha_{s}\right)$ corrections. The masses of the observed heavy-light mesons $\left(D, D^{*}\right),\left(D_{s}, D_{s}^{*}\right)$, ( $\left.D_{0}^{*}(2400), D_{1}(2430)\right),\left(D_{s 0}^{*}(2317), D_{s 1}(2460)\right),\left(B, B^{*}\right)$, $\left(B_{s}, B_{s}^{*}\right)$ can be well reproduced, while the predictions for the masses of $\left(B_{0}^{*}, B_{1}\right)$, and $\left(B_{s 0}^{*}, B_{s 1}\right)$ can be confronted with the experimental data in the futures. Up to the order $\mathcal{O}\left(\alpha_{S}\right)$, the QCD sum rules cannot lead to satisfactory values for $f_{D}, f_{D_{s}}$, and $f_{D_{s}} / f_{D}$ compared to the experimental data. We have to take into account the perturbative $\mathcal{O}\left(\alpha_{s}^{2}\right)$ corrections by choosing the pole masses, then the experimental data can be well reproduced. The present predictions for the decay constants of the heavy-light pseudoscalar, scalar, vector, and axial-vector mesons have many phenomenological applications in studying the semi-leptonic and leptonic decays of the heavy-light mesons.

Acknowledgments This work is supported by National Natural Science Foundation, Grant Numbers 11375063, and Natural Science Foundation of Hebei province, Grant Number A2014502017.

Open Access This article is distributed under the terms of the Creative Commons Attribution 4.0 International License (http://creativecomm ons.org/licenses/by/4.0/), which permits unrestricted use, distribution, and reproduction in any medium, provided you give appropriate credit to the original author(s) and the source, provide a link to the Creative Commons license, and indicate if changes were made. Funded by SCOAP ${ }^{3}$.

\section{References}

1. K.A. Olive et al., Chin. Phys. C 38, 090001 (2014)

2. J. Bordes, J. Penarrocha, K. Schilcher, JHEP 0412, 064 (2004)

3. J. Bordes, J. Penarrocha, K. Schilcher, JHEP 0511, 014 (2005)

4. S. Narison, Phys. Lett. B 718, 1321 (2013)

5. W. Lucha, D. Melikhov, S. Simula, Phys. Lett. B 701, 82 (2011)

6. W. Lucha, D. Melikhov, S. Simula, J. Phys. G 38, 105002 (2011)

7. P. Gelhausen, A. Khodjamirian, Al.A. Pivovarov, D. Rosenthal, Phys. Rev. D 88, 014015 (2013)

8. B. Blossier et al., JHEP 0907, 043 (2009)
9. C.T.H. Davies et al., Phys. Rev. D 82, 114504 (2010)

10. H. Na et al., Phys. Rev. D 86, 034506 (2012)

11. A. Bazavov et al., Phys. Rev. D 85, 114506 (2012)

12. S. Narison, Phys. Lett. B 668, 308 (2008)

13. Z.G. Wang, JHEP 1310, 208 (2013)

14. C.A. Dominguez, N. Paver, Phys. Lett. B 197, 423 (1987)

15. S. Narison, Phys. Lett. B 198, 104 (1987)

16. L.J. Reinders, Phys. Rev. D 38, 947 (1988)

17. M. Jamin, M. Munz, Z. Phys. C 60, 569 (1993)

18. S. Narison, Phys. Lett. B 520, 115 (2001)

19. M. Jamin, B.O. Lange, Phys. Rev. D 65, 056005 (2002)

20. H.Y. Jin, J. Zhang, Z.F. Zhang, Phys. Rev. D 81, 054021 (2010)

21. A. Hayashigaki, K. Terasaki, arXiv:hep-ph/0411285

22. Z.G. Wang, Chin. Phys. Lett. 25, 3908 (2008)

23. M.J. Baker, J. Bordes, C.A. Dominguez, J. Penarrocha, K. Schilcher, JHEP 1407, 032 (2014)

24. W. Lucha, D. Melikhov, S. Simula, Phys. Lett. B 735, 12 (2014)

25. W. Lucha, D. Melikhov, S. Simula, Phys. Rev. D 91, 116009 (2015)

26. Y.B. Dai, X.Q. Li, S.L. Zhu, Y.B. Zuo, Eur. Phys. J. C 55, 249 (2008)

27. T.M. Aliev, V.L. Eletsky, Sov. J. Nucl. Phys. 38, 936 (1983)

28. V.L. Eletsky, Phys. Atom. Nucl. 59, 2002 (1996)

29. K.G. Chetyrkin, M. Steinhauser, Phys. Lett. B 502, 104 (2001)

30. K.G. Chetyrkin, M. Steinhauser, Eur. Phys. J. C 21, 319 (2001)

31. A.A. Penin, M. Steinhauser, Phys. Rev. D 65, 054006 (2002)

32. S. Narison, Int. J. Mod. Phys. A 30, 1550116 (2015)

33. D. Becirevic et al., JHEP 1202, 042 (2012)

34. G.C. Donald, C.T.H. Davies, J. Koponen, G.P. Lepage, Phys. Rev. Lett. 112, 212002 (2014)

35. B. Colquhoun et al., Phys. Rev. D 91, 114509 (2015)

36. D. Becirevic et al., Phys. Rev. D 60, 074501 (1999)

37. G. Herdoiza, C. McNeile annd C. Michael. Phys. Rev. D 74, 014510 (2006)

38. E. Gamiz et al., Phys. Rev. D 80, 014503 (2009)

39. Z.G. Wang, W.M. Yang, S.L. Wan, Nucl. Phys. A 744, 156 (2004)

40. G. Cvetic, C.S. Kim, G.L. Wang, W. Namgung, Phys. Lett. B 596, 84 (2004)

41. D. Ebert, R.N. Faustov, V.O. Galkin, Phys. Lett. B 635, 93 (2006)

42. P. Colangelo, G. Nardulli, M. Pietroni, Phys. Rev. D 43, 3002 (1991)

43. M.Z. Yang, Eur. Phys. J. C 72, 1880 (2012)

44. A.M. Badalian, B.L.G. Bakker, Yu.A. Simonov, Phys. Rev. D 75, 116001 (2007)

45. H.M. Choi, Phys. Rev. D 75, 073016 (2007)

46. C.W. Hwang, Phys. Rev. D 81, 114024 (2010)

47. R.C. Verma, J. Phys. G 39, 025005 (2012)

48. X.H. Guo, M.H. Weng, Eur. Phys. J. C 50, 63 (2007)

49. D. Ebert, T. Feldmann, R. Friedrich, H. Reinhardt, Nucl. Phys. B 434, 619 (1995)

50. S. Nam, Phys. Rev. D 85, 034019 (2012)

51. S.S. Gershtein, M.Yu. Khlopov, JETP Lett. 23, 338 (1976)

52. M.A. Shifman, A.I. Vainshtein, V.I. Zakharov, Nucl. Phys. B 147, 385 (1979)

53. L.J. Reinders, H. Rubinstein, S. Yazaki, Phys. Rep. 127, 1 (1985)

54. Z.G. Wang, arXiv:1501.05093

55. Z.G. Wang, Phys. Rev. D 83, 014009 (2011)

56. P. Colangelo, F. De Fazio, F. Giannuzzi, S. Nicotri, Phys. Rev. D 86, 054024 (2012)

57. Z.G. Wang, Phys. Rev. D 88, 114003 (2013)

58. C. McNeile, C. Michael, Phys. Lett. B 642, 244 (2006)

59. K. Maltman, J. Kambor, Phys. Rev. D 65, 074013 (2002)

60. M. Diehl, G. Hiller, JHEP 06, 067 (2001)

61. H.Y. Cheng, F.S. Yu, Phys. Rev. D 89, 114017 (2014)

62. Z.G. Wang, Phys. Rev. D 77, 054024 (2008)

63. Z.G. Wang, Eur. Phys. J. C 56, 181 (2008)

64. Z.G. Wang, Commun. Theor. Phys. 52, 91 (2009) 
65. R.F. Dashen, Phys. Rev. 183, 1245 (1969)

66. Z.G. Wang, Phys. Rev. D 73, 094020 (2006)

67. Z.G. Wang, J. Phys. G 34, 753(2007)
68. Z.G. Wang, Phys. Rev. D 75, 034013 (2007)

69. A.G. Akeroyd, C.H. Chen, Phys. Rev. D 75, 075004 (2007)

70. A.G. Akeroyd, F. Mahmoudi, JHEP 0904, 121 (2009) 